\title{
Severe acute respiratory syndrome coronavirus 2 (SARS-CoV-
}

\section{2) antibody lateral flow assay for antibody prevalence studies}

\section{following vaccination: a diagnostic accuracy study [version 1;}

\section{peer review: 1 approved, 1 approved with reservations]}

\author{
Alexandra Cann (Di) $1,2^{*}$, Candice Clarke ${ }^{3,4^{*}}$, Jonathan Brown ${ }^{1 *}$, Tina Thomson (iD) 3,4 , \\ Maria Prendecki3,4, Maya Moshe (iD 3,4, Anjna Badhan1, Bryony Simmons ${ }^{5}$, \\ Bob Klaber², Paul Elliott(D1,2,6,7, Ara Darzi1,2,8, Steven Riley 1,7, Deborah Ashby (D1,6, \\ Paul Martin (103), Sarah Gleeson ${ }^{3}$, Michelle Willicombe3,4, Peter Kelleher ${ }^{1,9}$, \\ Helen Ward (D1,2,6-8, Wendy S. Barclay1', Graham S. Cooke (iD)1,2,8 \\ ${ }^{1}$ Department of Infectious Disease, Imperial College London, London, UK \\ ${ }^{2}$ Imperial College Healthcare NHS Trust, London, UK \\ ${ }^{3}$ Centre for Inflammatory Disease, Department of Immunology and Inflammation, Imperial College London, London, UK \\ ${ }^{4}$ Imperial College Renal and Transplant Centre, Imperial College Healthcare NHS Trust, London, UK \\ ${ }^{5}$ LSE Health, London School of Economics and Political Science, London, UK \\ ${ }^{6}$ School of Public Health, Imperial College London, London, UK \\ ${ }^{7} \mathrm{MRC}$ Centre for Global infectious Disease Analysis and Abdul Latif Jameel Institute for Disease and Emergency Analytics, Imperial \\ College London, London, UK \\ ${ }^{8}$ National Institute for Health Research, Imperial Biomedical Research Centre, London, UK \\ ${ }^{9}$ Chelsea \& Westminster NHS Trust, London, UK \\ ${ }^{*}$ Equal contributors
}

V1 First published: 21 Dec 2021, 6:358

https://doi.org/10.12688/wellcomeopenres.17231.1

Latest published: 26 May 2022, 6:358

https://doi.org/10.12688/wellcomeopenres.17231.2

\section{Abstract}

Background: Lateral flow immunoassays (LFIAs) are able to achieve affordable, large scale antibody testing and provide rapid results without the support of central laboratories. As part of the development of the REACT programme extensive evaluation of LFIA performance was undertaken with individuals following natural infection. Here we assess the performance of the selected LFIA to detect antibody responses in individuals who have received at least one dose of severe acute respiratory syndrome coronavirus 2 (SARSCoV-2) vaccine.

Methods: This was a prospective diagnostic accuracy study. Sampling was carried out at renal outpatient clinic and healthcare worker testing sites at Imperial College London NHS Trust. Two cohorts of patients were recruited; the first was a cohort of 108 renal transplant

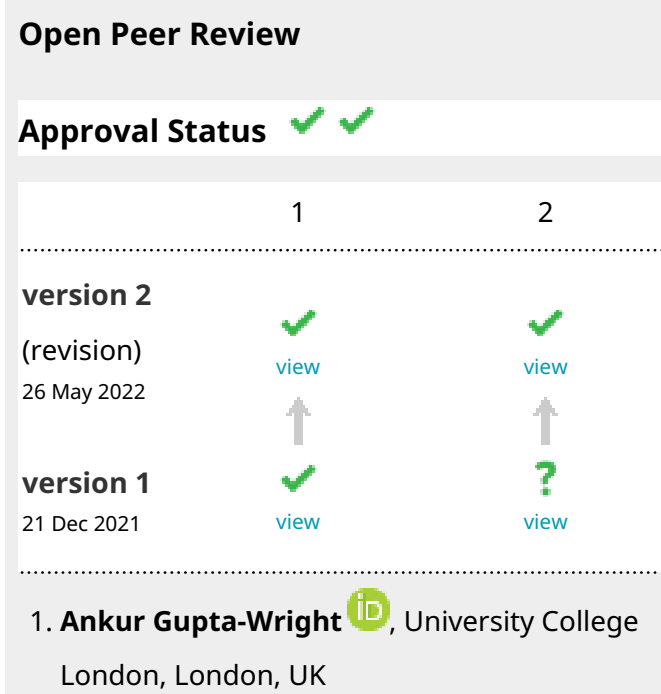

London, London, UK 
patients attending clinic following two doses of SARS-CoV-2 vaccine, the second cohort comprised 40 healthcare workers attending for first SARS-CoV-2 vaccination and subsequent follow up. During the participants visit, finger-prick blood samples were analysed on LFIA device, while paired venous sampling was sent for serological assessment of antibodies to the spike protein (anti-S) antibodies. AntiS IgG was detected using the Abbott Architect SARS-CoV-2 IgG Quant II CMIA. A total of 186 paired samples were collected. The accuracy of Fortress LFIA in detecting IgG antibodies to SARS-CoV-2 compared to anti-spike protein detection on Abbott Assay

Results: The LFIA had an estimated sensitivity of 92.0\% (114/124; 95\% confidence interval [CI] $85.7 \%$ to $96.1 \%$ ) and specificity of $93.6 \%$ (58/62; 95\% CI 84.3\% to 98.2\%) using the Abbott assay as reference standard (using the threshold for positivity of $7.10 \mathrm{BAU} / \mathrm{ml}$ )

Conclusions: Fortress LFIA performs well in the detection of antibody responses for intended purpose of population level surveillance but does not meet criteria for individual testing.

Keywords

SARS-CoV-2, Covid-19, Lateral flow, LFIA, Antibodies, Neutralisation, Seroprevalence

This article is included in the Coronavirus

(COVID-19) collection.

\section{Hans-Michael Kaltenbach ID, ETH Zürich,}

Basel, Switzerland

Any reports and responses or comments on the article can be found at the end of the article. 


\section{Corresponding authors: Alexandra Cann (alexandra.cann@nhs.net), Graham S. Cooke (g.cooke@imperial.ac.uk)}

Author roles: Cann A: Conceptualization, Data Curation, Formal Analysis, Investigation, Methodology, Project Administration, Writing Original Draft Preparation; Clarke C: Data Curation, Investigation, Methodology, Project Administration, Resources, Writing - Review \& Editing; Brown J: Data Curation, Formal Analysis, Investigation, Methodology, Writing - Review \& Editing; Thomson T: Data Curation, Formal Analysis, Investigation, Methodology, Writing - Review \& Editing; Prendecki M: Data Curation, Formal Analysis, Investigation, Methodology, Writing - Review \& Editing; Moshe M: Data Curation, Formal Analysis, Investigation, Methodology, Writing - Review \& Editing; Badhan A: Writing - Review \& Editing; Simmons B: Formal Analysis, Methodology, Validation, Writing - Review \& Editing; Klaber B: Writing - Review \& Editing; Elliott P: Writing - Review \& Editing; Darzi A: Writing - Review \& Editing; Riley S: Writing - Review \& Editing; Ashby D: Writing - Review \& Editing; Martin P: Data Curation, Investigation, Writing - Review \& Editing; Gleeson S: Investigation, Methodology, Writing - Review \& Editing; Willicombe M: Conceptualization, Data Curation, Investigation, Methodology, Supervision, Writing - Review \& Editing; Kelleher P: Writing - Review \& Editing; Ward H: Conceptualization, Investigation, Methodology, Resources, Writing - Review \& Editing; Barclay WS: Conceptualization, Data Curation, Investigation, Methodology, Resources, Supervision, Writing - Review \& Editing; Cooke GS: Resources, Supervision, Writing - Review \& Editing

Competing interests: No competing interests were disclosed.

Grant information: The study was funded by the Department of Health and Social Care in England. LFIA devices were provided free of charge for research purposes. HW is a National Institute for Health Research (NIHR) Senior Investigator and acknowledges support from NIHR Biomedical Research Centre of Imperial College NHS Trust, NIHR School of Public Health Research, NIHR Applied Research Collaborative North West London, and Wellcome [205456, https://doi.org/10.35802/205456]. GC is supported by an NIHR Professorship. $A D$ is an NIHR senior investigator and DA and PE are Emeritus NIHR Senior Investigators. SR acknowledges support from MRC Centre for Global Infectious Disease Analysis, National Institute for Health Research (NIHR) Health Protection Research Unit (HPRU), Wellcome [200861, https://doi.org/10.35802/200861, 200187, https://doi.org/10.35802/200187], and Centres for Disease Control and Prevention [US, U01CK0005-01-02]. PE is Director of the MRC Centre for Environment and Health [MR/L01341X/1, MR/S019669/1]. PE acknowledges support from the NIHR Imperial Biomedical Research Centre and the NIHR HPRUs in Chemical and Radiation Threats and Hazards and in Environmental Exposures and Health, the British Heart Foundation Centre for Research Excellence at Imperial College London [RE/18/4/34215], Health Data Research UK (HDR UK) and the UK Dementia Research Institute at Imperial [MC_PC_17114]. The funders had no role in study design, data collection and analysis, decision to publish, or preparation of the manuscript.

Copyright: ( $) 2021$ Cann A et al. This is an open access article distributed under the terms of the Creative Commons Attribution License, which permits unrestricted use, distribution, and reproduction in any medium, provided the original work is properly cited.

How to cite this article: Cann A, Clarke C, Brown J et al. Severe acute respiratory syndrome coronavirus 2 (SARS-CoV-2) antibody lateral flow assay for antibody prevalence studies following vaccination: a diagnostic accuracy study [version 1; peer review: 1 approved, 1 approved with reservations] Wellcome Open Research 2021, 6:358 https://doi.org/10.12688/wellcomeopenres.17231.1

First published: 21 Dec 2021, 6:358 https://doi.org/10.12688/wellcomeopenres.17231.1 


\section{Introduction}

As vaccination programmes for coronavirus disease 2019 (COVID-19) are rolled out worldwide, population antibody testing is useful in monitoring immune responses to vaccinations, informing discussion and decisions about booster doses, and assessing levels of potential protective immunity in the population ${ }^{1}$.

Lateral flow immunoassays (LFIAs) have the potential to deliver affordable, large-scale testing of individuals and provide rapid results without the support of central laboratories Antigen lateral flow testing is already being used widely. This approach, using antibody lateral flow devices, has been used across England in the REACT2 (REal time Assessment of Community Transmission) ${ }^{2}$ study to estimate the number of infections during the first wave of the COVID-19 pandemic ${ }^{2}$, monitor the decline in antibody positivity over time ${ }^{3}$ and assess population antibody prevalence following vaccine roll-out, most recently in Round 5 of the study published in February $2021^{4}$.

Prior to the scale up of antibody testing for surveillance, extensive clinical and laboratory evaluation of diagnostic accuracy following natural infection was performed on a range of LFIA antibody tests ${ }^{5,6}$, identifying one for subsequent use. The test selected (Fortress, Northern Ireland) detects antibody against the spike protein of the virus (contained in all licensed vaccines) and would therefore be expected to detect vaccine induced antibody responses. This study examined the accuracy of the Fortress LFIA device in detecting antibodies in two cohorts of vaccinated individuals and explored the relationship between LFIA results and viral neutralisation.

\section{Methods}

This was a prospective diagnostic accuracy study conducted between 20th December 2020 and $26^{\text {th }}$ May 2021. Samples were collected from two groups: renal transplant patients (cohort 1) and healthcare workers (cohort 2).

\section{Bias}

Every attempt was made to address potential sources of bias. All eligible participants were offered enrolment where practical and every effort was made to ensure understanding of the participant information sheet (PIS) and study procedure, using translation services where necessary. Potential participants were given time to consider participation and trained research staff were able to answer questions relating to the study.

\section{Eligibility criteria}

Eligibility for both cohorts was defined as:

1) Adult $(>/=18$ years old $)$

2) Able to understand and consent to study

3) Received either one or two doses of any UK approved vaccine for COVID-19

4) Able to comply with study procedure/ study not thought to be risk to patient
Sample size

Sample size was computed based on an expected sensitivity of $90 \%$ and specificity of $95 \%$, with a minimal acceptable lower confidence limit of $-10 \%$ for both estimates. Under power $1-\beta=0.85$ and $\alpha=0.15$, the minimum sample size required is 106 cases and 76 controls. Patients were pragmatically enrolled to ensure minimum sample size achieved.

\section{Cohort 1: Renal transplant cohort}

Participants. Participants were recruited between $1^{\text {st }}$ February 2021 and $26^{\text {th }}$ May 2021.Those recruited were 108 renal transplant recipients who were attending clinic at Hammersmith Hospital 28 days (allowing range from 21 to 42 days) following a second dose of a SARS-CoV-2 vaccine, (either BNT162b2, Pfizer/BioNTech or ChAdOx1, Oxford/AstraZeneca). Participants were recruited directly from clinic by trained medical and nursing staff who explained the study and provided with PIS and informed consent form (ICF). Participants underwent a finger-prick capillary blood draw for immediate testing on the LFIA device and, at the same time, gave a venous blood samples for later serology testing. Clinical characteristics were obtained from electronic medical records (including basic demographic data, past medical history, vaccination status; see Table 1 for full details). All patients provided written informed consent.

Lateral flow immunoassay testing. Participants were supplied with an LFIA testing kit as used in the REACT home testing programme ${ }^{7}$. The LFIA (Fortress, NI) detects IgG and IgM to the S1 subunit of the spike protein. Participants were also provided with verbal instructions on how to use the test by a member of the research team, prior to performing self-testing, with support provided where necessary. The LFIA result was assessed independently by two observers. The results were reported by the colour intensity of the IgG band, and documented as either a positive or negative result.

\section{Cohort 2: Healthcare worker cohort}

Participants. Participants were recruited between $20^{\text {th }}$ December 2020 and $31^{\text {st }}$ January 2021. Overall, 39 healthcare workers were consented when attending for first vaccination with BNT162b2 Pfizer-BioNTech. Participants were approached by trained members of the research team at the vaccination centre and provided with a PIS and ICF with explanation of the study. Of these participants, 38 had repeat samples taken at 21 days post vaccination and one further participant had samples taken at 21 days who had not had day 0 samples. In total there were 40 participants. Data was collected on age and gender. Medical records of participants were not accessed for this cohort.

Lateral flow immunoassay testing. Participants were supported in capillary blood draw for use with the Fortress LFIA devices as described above. Results were reviewed and recorded by the study team.

Serological testing. Serological assessment was performed on the Abbott Architect SARS-CoV-2 IgG Quant II CMIA which 
Table 1. Clinical characteristics renal transplant cohort. Note table refers to Abbott serology result. Subsequently, one seronegative individual tested positive using DABA.

\begin{tabular}{|c|c|c|c|c|}
\hline \multicolumn{2}{|l|}{ Characteristic } & \multirow{2}{*}{ 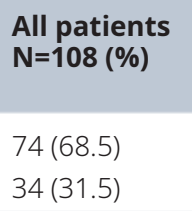 } & \multirow{2}{*}{$\begin{array}{l}\text { Anti-S } \\
\text { Seronegative* } \\
\mathbf{N =} \mathbf{3 6}(\%) \\
25(69.4) \\
11(30.6)\end{array}$} & \multirow{2}{*}{$\begin{array}{l}\begin{array}{l}\text { Anti-S } \\
\text { Seropositive* } \\
\text { N= } 72 \text { (\%) }\end{array} \\
49(68.1) \\
23(31.9)\end{array}$} \\
\hline Gender & $\begin{array}{l}\text { Male } \\
\text { Female }\end{array}$ & & & \\
\hline Age & Years (Range) & $54(41-65)$ & $44(38-74)$ & $56(44-64)$ \\
\hline Ethnicity & $\begin{array}{l}\text { White } \\
\text { Black } \\
\text { Asian } \\
\text { Other }\end{array}$ & $\begin{array}{l}52(48.1) \\
8(7.4) \\
34(31.5) \\
14(13.0)\end{array}$ & $\begin{array}{l}17(47.2) \\
2(5.6) \\
11(30.6) \\
6(16.7)\end{array}$ & $\begin{array}{l}35(48.6) \\
6(8.3) \\
23(31.9) \\
8(11.1)\end{array}$ \\
\hline Cause of End Stage Kidney Disease & $\begin{array}{l}\text { Polycystic kidney disease } \\
\text { Glomerulonephritis } \\
\text { Diabetic nephropathy } \\
\text { Urological } \\
\text { Unknown } \\
\text { Other }\end{array}$ & $\begin{array}{l}9(8.3) \\
41(38.0) \\
18(16.7) \\
7(6.5) \\
26(24.1) \\
7(6.5)\end{array}$ & $\begin{array}{l}4(11.1) \\
12(33.3) \\
7(19.4) \\
2(5.6) \\
8(22.2) \\
3(8.3)\end{array}$ & $\begin{array}{l}5(6.9) \\
29(40.3) \\
11(15.3) \\
5(6.9) \\
18(25.0) \\
4(5.6)\end{array}$ \\
\hline Vaccinated $\leq 1$ year post transplant & $\begin{array}{l}\text { Yes } \\
\text { No }\end{array}$ & $\begin{array}{l}6(5.6) \\
102(94.4)\end{array}$ & $\begin{array}{l}1(2.8) \\
35(97.2)\end{array}$ & $\begin{array}{l}5(6.9) \\
67(93.1)\end{array}$ \\
\hline Time vaccinated post-transplant & Years (Median) & $6.3(2.9-11.9)$ & $5.7(2.8-11.7)$ & $6.5(2.9-12.0)$ \\
\hline Diabetes & $\begin{array}{l}\text { No } \\
\text { Yes }\end{array}$ & $\begin{array}{l}75(69.4) \\
33(30.6)\end{array}$ & $\begin{array}{l}27(75.0) \\
9(25.0)\end{array}$ & $\begin{array}{l}48(66.7) \\
24(33.3)\end{array}$ \\
\hline Vaccine type & $\begin{array}{l}\text { BNT162b2 } \\
\text { ChAdOx1 }\end{array}$ & $\begin{array}{l}51(47.2) \\
57(52.8)\end{array}$ & $\begin{array}{l}12(33.3) \\
24(66.7)\end{array}$ & $\begin{array}{l}39(54.2) \\
33(45.8)\end{array}$ \\
\hline Time between vaccinations & Days (median) & $77(73-80)$ & $77(71-80)$ & $77(74-80)$ \\
\hline Time of serological test post-boost & Days (median) & $34(29-38)$ & $34(29-36)$ & $34(30-38)$ \\
\hline Prior COVID-19 exposure & $\begin{array}{l}\text { No } \\
\text { Yes }\end{array}$ & $\begin{array}{l}89(82.4) \\
19(17.6)\end{array}$ & $\begin{array}{l}36(100.0) \\
-\end{array}$ & $\begin{array}{l}53(73.6) \\
19(26.4)\end{array}$ \\
\hline
\end{tabular}

reports a quantitative anti-Spike antibody titre. The threshold value for positivity of 7.10 binding antibody units (BAU)/ $\mathrm{ml}$. At the time of the study in the healthcare worker cohort (cohort 2), quantitative antibody titres were reported in AU/ml. To allow combination with cohort 1 data, these were converted to $\mathrm{BAU} / \mathrm{ml}$ by multiplying by 0.142 . Double antigen binding assay (DABA) testing for discordant results (positive LFIA with negative serological) was performed on available stored samples from cohort 1. Detailed methodology of DABA has been described previously. Briefly, the Imperial Hybrid DABA is a sequential two step double binding assay for the detection and measurement of antibody directed to the receptor binding domain of SARS-CoV-2. The proteins employed were expressed and gifted by the Crick Institute, London. In order to evaluate specificity the Hybrid DABA was tested on stored plasma and serum samples predating the SARS-CoV-2 outbreak $(\mathrm{n}=825)$ in which 0 samples tested positive, giving a specificity of $100 \%$.

In addition, for cohort 2 , individuals provided blood for assessment of neutralisation assays. The ability of sera to neutralise the SARS-CoV-2 virus was assessed by neutralisation assay on Vero cells. Sera were serially diluted in OptiPRO SFM (Life Technologies) and incubated for $1 \mathrm{~h}$ at room temperature with 100 TCID50/well of SARS-CoV-2/England/IC19/2020 and transferred to 96-well plates pre-seeded with Vero-E6 cells. Serum dilutions were performed in duplicate. Plates were incubated at $37^{\circ} \mathrm{C}, 5 \% \mathrm{CO}_{2}$ for $42 \mathrm{~h}$ before fixing cells in $4 \%$ PFA (paraformaldehyde). Cells were treated with methanol $0.6 \%$ $\mathrm{H}_{2} \mathrm{O}_{2}$ and stained for $1 \mathrm{~h}$ with a 1:3000 dilution of 40143R019 rabbit $\mathrm{mAb}$ to SARS-CoV-2 nucleocapsid protein (Sino Biological). A 1:3000 dilution of sheep anti-rabbit HRP 
(horseradish peroxidase) conjugate (Sigma) was then added for 1 h. TMB (3,3',5,5'-Tetramethylbenzidine) substrate (Europa Bioproducts) was added and developed for 20 mins before stopping the reaction with $1 \mathrm{M}$ hydrogen chloride $(\mathrm{HCl})$. Plates were read at $450 \mathrm{~nm}$ and $620 \mathrm{~nm}$ and the concentration of serum needed to reduce virus signal by $50 \%$ was calculated to give NT50 values.

\section{Performance analysis}

The primary outcome of the study was sensitivity and specificity of the LFIA device in detecting SARS-CoV-2 IgG antibodies identified by the Abbott platform.

A secondary analysis was conducted using reference standard as either Abbott or, for discordant results (positive LFIA negative serology) in cohort 1 using in house DABA as reference standard for serological positivity.

Outcomes are presented with the corresponding binomial exact 95\% confidence interval (95\% CI). Statistical analyses (specificity and sensitivity) were performed with open access online website MedCalc diagnostic test evaluation calculator (version 20.015). Graphical analyses was performed using GraphPad Prism 9.1.2 software. An open-source alternative is R.

\section{Ethics approval}

Ethics approvals were sought for each cohort prior to commencement of the study.

The renal cohort ethics were obtained from Health Research Authority, Research Ethics Committee (Reference: 20/WA/0123 - The Impact of COVID-19 on Patients with Renal disease and Immunosuppressed Patient).

For the healthcare worker cohort, we got ethics from the South Central-Berkshire B Research Ethics Committee (IRAS ID: 283805), and Medicines and Healthcare products Regulatory Agency approval for use of the LFIA for research purposes only.

\section{Results}

\section{Cohort characteristics}

The characteristics of the participants are described in Table 1 and Table 2. In total, 186 samples were tested using both LFIA and serological testing 9 .

\section{LFIA IgG positivity and antibody titres in serum}

The combined results describe both cohort 1 and cohort 2 ( $n=186$ samples, Figure 1). Of those samples which scored positive on LFIA ( $\mathrm{n}=118), 4$ had undetectable laboratory anti$S$ levels using Abbott Architect assay. Three of these samples (from the renal transplant cohort) were subsequently re-tested using an in-house DABA which detected antibodies in 1 sample and confirmed negativity in 2 . The remaining 114 samples had a median anti-S titre of $229.5 \mathrm{BAU} / \mathrm{ml}$ and mean of $229.5 \mathrm{BAU} / \mathrm{ml}$; anti-S titre ranged from $9.7 \mathrm{BAU} / \mathrm{ml}$ to 5680 BAU $/ m$ l. Of those which scored negative on LFIA $(n=68)$, anti-S antibodies were detected in 10 samples, of which 7 had anti-S titre levels <10 BAU/ml (7.8, 8.0, 8.5, 8.8, 9.2, 9.4, 9.7). The other 58 negative LFIA tests had undetectable anti-S levels $(<7.1 \mathrm{BAU} / \mathrm{ml})$.

\section{Test sensitivity and specificity}

Primary analysis (Abbott as reference standard). Using the threshold value for positivity on serological testing of $\geq 7.10$ $\mathrm{BAU} / \mathrm{ml}$, the LFIA had an estimated sensitivity of $92.0 \%$ $(114 / 124 ; 95 \%$ CI $85.7 \%$ to $96.1 \%)$ and specificity of $93.6 \%$ $(58 / 62 ; 95 \%$ CI $84.3 \%$ to $98.2 \%)$ using the Abbott assay as reference standard.

Secondary analysis (Abbott or DABA as reference standard). Using the threshold vale for positivity on serological testing of $\geq 7.10 \mathrm{BAU} / \mathrm{ml} \quad(\mathrm{n}=183)$ on Abbott Architect Assay and confirmatory DABA result for available discordant samples $(n=3)$ as the reference standard, the overall performance of the test in these combined cohorts produce an estimate of sensitivity

Table 2. Clinical characteristics of healthcare worker cohort.

\begin{tabular}{|c|c|c|c|c|c|c|}
\hline \multirow{2}{*}{ Characteristic } & & \multirow{2}{*}{$\begin{array}{l}\text { All patients } \\
\mathrm{N}=40(\%)\end{array}$} & \multicolumn{2}{|c|}{$\begin{array}{l}\text { Anti-S } \\
\text { Seronegative }\end{array}$} & \multicolumn{2}{|c|}{ Anti-S Seropositive } \\
\hline & & & $\begin{array}{l}\mathrm{D0} \\
\mathrm{N}=26(\%)\end{array}$ & $\begin{array}{l}\mathrm{D} 21 \\
\mathrm{~N}=0 \\
(\%)\end{array}$ & $\begin{array}{l}\mathrm{DO} \\
\mathrm{N}=13 \\
(\%)\end{array}$ & $\begin{array}{l}\mathrm{D} 21 \\
\mathrm{~N}=39 \\
(\%)\end{array}$ \\
\hline Gender & $\begin{array}{l}\text { Male } \\
\text { Female }\end{array}$ & $\begin{array}{l}13(32.5) \\
27(67.5)\end{array}$ & $\begin{array}{l}10(38.5) \\
16(61.5)\end{array}$ & $\begin{array}{l}0 \\
0\end{array}$ & $\begin{array}{l}3(23.0) \\
10(77.0)\end{array}$ & $\begin{array}{l}13(33.3) \\
27(66.6)\end{array}$ \\
\hline Age & Years average (range) & $43(23-71)$ & 46 & - & 38 & 43 \\
\hline Vaccine type & BNT162b2 & $40(100)$ & \multicolumn{4}{|l|}{-} \\
\hline Time between vaccinations & Days average (range) & 65 (57-92) & $\begin{array}{l}- \\
-\end{array}$ & & & \\
\hline
\end{tabular}




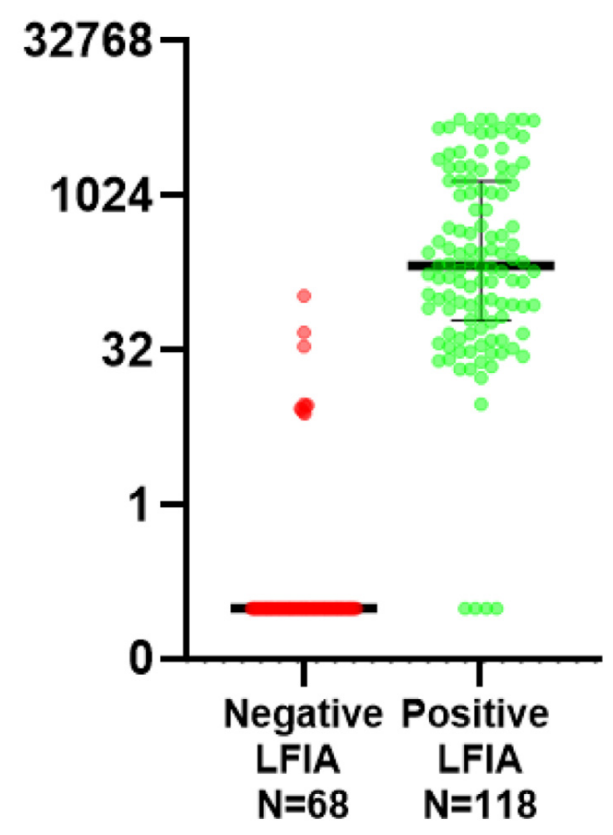

Figure 1. Paired Quantitative anti-S titres (Abbott) between those testing positive and negative on Fortress lateral flow immuno-assay (LFIA) for combined cohorts 1 and 2. (Y-axis uses Log 2 scale, data points below the lower limit of detection are assigned arbitrary value of 0.01 for plotting).

of $92.0 \%(115 / 125 ; 95 \%$ CI $85.8 \%$ to $96.1 \%)$ and specificity of $95.1 \%(58 / 61 ; 95 \%$ CI $86.3 \%$ to $99.0 \%)$. Results were similar when analysing cohort 1 and cohort 2 individually (see Figure 2).

\section{Live virus neutralisation}

Neutralisation titres were available for 64/78 samples in the healthcare worker cohort. Neutralisation titres (NT50) were significantly higher in those with positive LFIA compared to those without (Figure 3a). Only one LFIA-negative sample had detectable neutralisation assay using a threshold for positivity of (NT50 of 15 with an anti-S antibody titre of $7.8 \mathrm{BAU} / \mathrm{ml}$ ). For individuals with detectable $\operatorname{IgG}$ on LFIA only $2 / 34$ did not have significant evidence of viral neutralisation.

\section{Discussion}

This study demonstrates that the Fortress LFIA device performs well in detecting IgG antibodies in vaccinated individuals when comparing against a serological assay widely used in routine practice. LFIAs have been a helpful tool the assessment of population antibody prevalence of SARS-CoV-2, and can play a role in informing vaccination strategy going forwards. The Fortress LFIA has been assessed previously for its performance following natural infection ${ }^{6}$, though did not meet Medicines and Healthcare products Regulatory Agency (MHRA) criteria for individual use which recommend antibody tests should have a sensitivity of $>98 \%$ (95\% CI $96 \%$ to $100 \%$ ) and specificity of $>98 \%$ on a minimum of 200 known negative controls ${ }^{10}$. The test has undergone extensive evaluation for home self-testing ${ }^{7}$ and has since been used widely in community studies of antibody prevalence in England.

The performance of the LFIA in the cohorts of vaccinated individuals here demonstrates slightly higher sensitivity than previously reported for natural infection, though this difference is not significant. This is likely to reflect higher background titres of antibody following vaccination, particularly after second doses, when compared to natural infection in the community, at least in the healthcare worker cohort. The LFIA device does not detect very low levels of antibody which may still correlate with protection from severe disease and/ or hospitalisation. However, in the general population, the number of such individuals with low titres following two vaccinations will be low (in contrast to the renal transplant cohort studied here).

A small number of LFIA tests appear to produce false positive results $(n=4)$ with undetectable antibodies in the commercial laboratory assay. To understand whether these were genuine false positives, these four samples were tested with a second sensitive assay (DABA). Only one if these discordant samples tested positive.

There is growing evidence that the presence of neutralising antibodies in sera is highly predictive of protection from symptomatic COVID-19 disease ${ }^{11,12}$. Although the LFIA studied has a threshold below which it can't detect Spike specific antibody that is present, that threshold is close to the level at which neutralising antibody can be reliably measured (Figure 3a). This suggests that antibody positivity on the LFIA may give some indication of protection from symptomatic disease and thus could be useful to measure any waning of vaccine induced immunity in different populations.

In terms of study limitations, it is important to note that, although the LFIAs were self-tested in the clinic or vaccination centre, participants had access to support from trained healthcare professionals when required. This does not fully replicate the 'real-world' application of LFIAs where users will be following a detailed guide in their own homes. Furthermore, the patient cohort includes healthcare workers and as such may have greater understanding and/or experience of self-testing than members of the general population. However, the primary purpose of this study was to evaluate the diagnostics accuracy of the test.

The performance of the LFIA evaluated is sufficiently good that it can continue to play a helpful role in the assessment of population antibody responses resulting from widespread infection and high levels vaccination coverage, particularly given the correlation of LFIA results with the functional measure of live virus neutralisation. Over time, antibody titres will begin to wane and ongoing population surveillance can play a helpful role in informing decisions on policy for subsequent vaccination programmes, the targeting of booster vaccines. Rapid antibody testing may prove useful in initial screening of patients to receive monoclonal antibody therapy as lab methods may cause a delay in therapy to potentially eligible patients. 


\section{Renal Transplant Cohort (Cohort} 1; $n=108$ )

\section{Healthcare Workers Cohort} (Cohort 2; n=78)

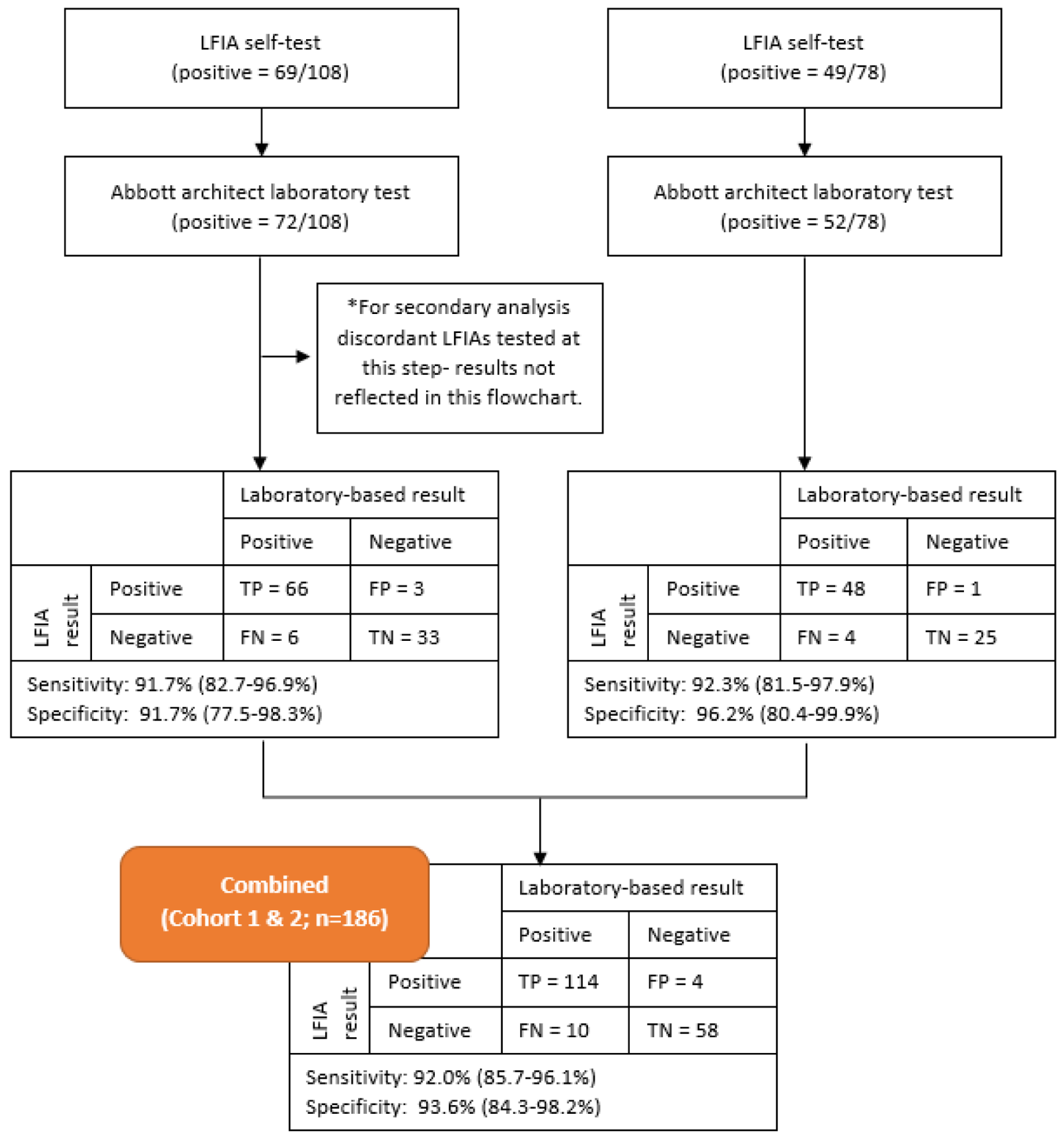

Figure 2. Flowchart detailing testing results for Cohort 1 and Cohort $\mathbf{2}$ and Combined Cohort. LFIA=Fortress lateral flow immuno-assay. 
A)

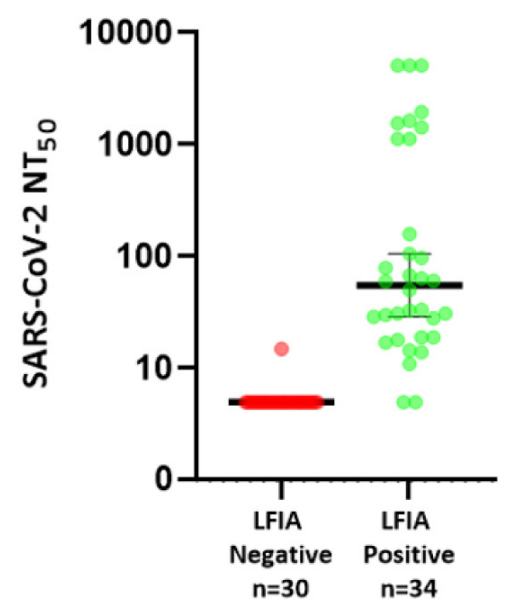

B)

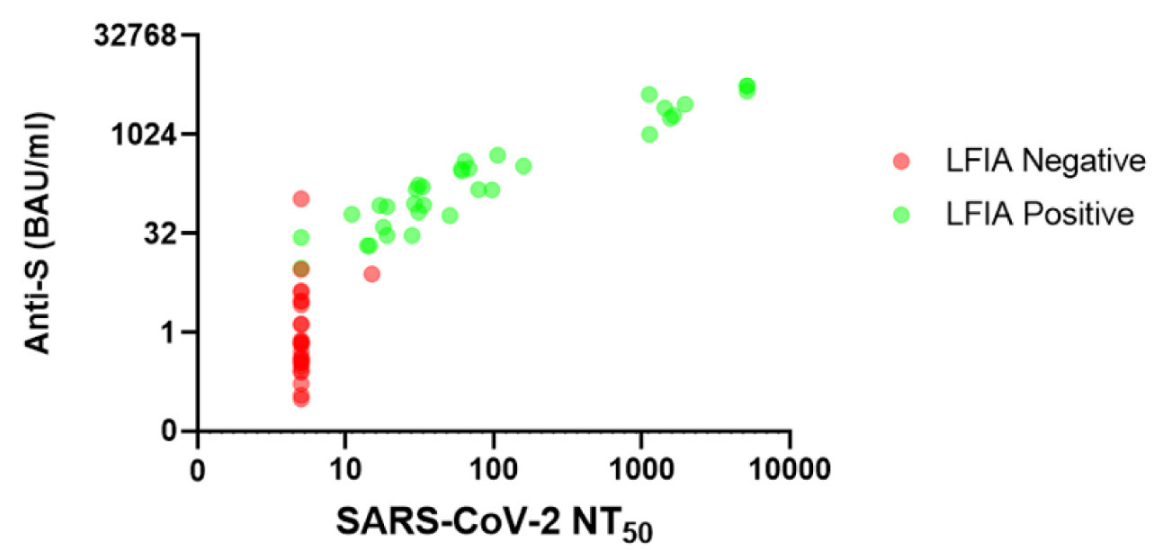

Figure 3. (A) Distribution of NT50 (neutralisation titre) values for individuals within cohort 2 according to whether positive or negative by Fortress lateral flow immuno-assay (LFIA) (Y-axis uses Log 10 scale, data points below the lower limit of detection are assigned arbitrary value of 0.01 for plotting) (B) Relationship between neutralisation titre and anti-S titre in Abbott assay (green representing those LFIA positive, red LFIA negative). (Y-axis uses Log 2 scale, data points below the lower limit of detection are assigned arbitrary value of 0.01 for plotting). SARS-CoV-2 NT=severe acute respiratory syndrome coronavirus 2 neutralisation titre.

\section{Data availability \\ Underlying data}

Harvard Dataverse Repository: "Replication Data for: SARS-CoV-2 Antibody Lateral Flow Assay for Possible Use in Seroprevalence Surveys: a Diagnostic Accuracy Study", https://doi.org/10.7910/DVN/KCDZIN9.

This project contains the following underlying data:

- Anti Spike Protein LFIA For HCW Cohort.tab
- Anti Spike Protein LFIA For Renal Cohort.tab

- Neutralisation Titres for HCW Cohort.tab

- DABA testing of LFIA Positive Abbott Negative Renal Transplant Samples.tab

Data are available under the terms of the Creative Commons Zero "No rights reserved" data waiver (CC0 1.0 Public domain dedication).
1. Maple PAC: Population (Antibody) Testing for COVID-19-Technical Challenges, Application and Relevance, an English Perspective. Vaccines (Basel). 2021; 9(6): 550.

PubMed Abstract | Publisher Full Text | Free Full Text

2. Ward $\mathrm{H}$, Atchison C, Whitaker M, et al.: Antibody prevalence for SARS-CoV-2 in England following first peak of the pandemic: REACT2 study in 100,000 adults. bioRxiv. 2020.

3. Ward H, Cooke G, Atchison C, et al.: Declining prevalence of antibody positivity to SARS-CoV-2: a community study of 365,000 adults. Cold Spring Harbor Laboratory. 2020

Publisher Full Text

4. Ward H, Cooke G, Whitaker M, et al.: REACT-2 Round 5: increasing prevalence of SARS-CoV-2 antibodies demonstrate impact of the second wave and of vaccine roll-out in England. Cold Spring Harbor Laboratory; 2021. Publisher Full Text

5. Flower B, Brown JC, Simmons B, et al.: Clinical and laboratory evaluation of SARS-CoV-2 lateral flow assays for use in a national COVID-19 seroprevalence survey. Thorax. 2020; 75(12): 1082-1088. PubMed Abstract | Publisher Full Text | Free Full Text

6. Moshe M, Daunt A, Flower B, et al.: SARS-CoV-2 lateral flow assays for possible use in national covid-19 seroprevalence surveys (React 2): diagnostic accuracy study. BMJ. 2021; 372: n423. PubMed Abstract | Publisher Full Text | Free Full Text
7. Atchison C, Pristerà P, Cooper E, et al.: Usability and Acceptability of Homebased Self-testing for Severe Acute Respiratory Syndrome Coronavirus 2 (SARS-CoV-2) Antibodies for Population Surveillance. Clin Infect Dis. 2021; 72(9): e384-e393.

PubMed Abstract | Publisher Full Text | Free Full Text

8. Flower B, Brown JC, Simmons B, et al.: Clinical and laboratory evaluation of SARS-CoV-2 lateral flow assays for use in a national COVID-19 seroprevalence survey. Thorax. 2020; 75(12): 1082-1088. PubMed Abstract | Publisher Full Text | Free Full Text

9. Cann A: Replication Data for: SARS-CoV-2 Antibody Lateral Flow Assay for Possible Use in Seroprevalence Surveys: a Diagnostic Accuracy Study. Harvard Dataverse, V1. UNF:6:VAXtmGNrXcnYiw/LspmAzQ== [fileUNF]. 2021. http://www.doi.org/10.7910/DVN/KCDZIN

10. MHRA: Target product profile antibody tests to help determine if people have immunity to SARS-CoV-2. [Accessed 21.6.21] Reference Source

11. Khoury DS, Cromer D, Reynaldi A, et al.: Neutralizing antibody levels are highly predictive of immune protection from symptomatic SARS-CoV-2 infection. Nat Med. Springer Science and Business Media LLC; 2021; 27(7): 1205-1211. PubMed Abstract | Publisher Full Text

12. Earle KA, Ambrosino DM, Fiore-Gartland A, et al.: Evidence for antibody as a protective correlate for COVID-19 vaccines. Vaccine. Cold Spring Harbor Laboratory; 2021; 39(32): 4423-4428. PubMed Abstract | Publisher Full Text | Free Full Text 


\section{Open Peer Review}

\section{Current Peer Review Status:}

\section{Version 1}

Reviewer Report 11 February 2022

https://doi.org/10.21956/wellcomeopenres.19045.r48522

(C) 2022 Kaltenbach $\mathbf{H}$. This is an open access peer review report distributed under the terms of the Creative Commons Attribution License, which permits unrestricted use, distribution, and reproduction in any medium, provided the original work is properly cited.

\section{Hans-Michael Kaltenbach}

Department of Biosystems Science and Engineering, Swiss Institute of Bioinformatics (SIB), ETH Zürich, Basel, Switzerland

This study determines the diagnostic accuracy of the Fortress LFIA for SARS-CoV-2 IgG antibodies after vaccination against an established reference assay. Specificity and sensitivity are estimated from samples of two cohorts and pooled estimates are reported as the main result. The authors provide an in-depth analysis of false-positive results and report additional results of neutralisation assays for one cohort. The results show lower sensitivity and higher specificity compared to the two cited previous evaluations of this LFIA, but both differences are not statistically significant. Overall, the study is well documented and the availability of raw data allows easy reproduction of the results.

My only concern is the sample design and analysis of the HCW cohort. The calculations assume that the 78 samples are independent, but they are in fact pairs from 39 individuals measured before and after vaccination. I would sympathize with neglecting this issue for cases with a negative result at day 0 followed by a positive result at day 21 , which one might treat as 'sufficiently' independent; however, one is not surprised to find the 13 positive samples of day 0 to also test positive at day 21. An ad-hoc remedy for this double-counting is to remove 13 of these 26 samples from the calculation as they provide no new information and artificially inflate the sample size. The estimated sensitivity is then reduced to $87.7 \%$ with a wider $95 \%$-CI of $[75.8,97.1]$ (compare to the reported 92.3\%) for that cohort, and to $91.0 \%$ (95\%-CI [84.1, 95.6]) instead of $92.0 \%$ for the pooled analysis. Specificities are of course unaffected. The equally problematic case of negative results on both occasions does not occur in these data. Please comment on the sample design of the HCW cohort and its consequences.

Three minor corrections:

A significance level of $15 \%$ is reported for the sample size calculation; statistical folklore and the reported sample size suggest it to mean $5 \%$.

In Table 2, you report $\mathrm{N}=39$ for the $\mathrm{D} 21$ seropositive column, but males and females add to 
40.

Please add a label for the vertical axis of Figure 1.

Is the work clearly and accurately presented and does it cite the current literature? Yes

Is the study design appropriate and is the work technically sound?

Yes

Are sufficient details of methods and analysis provided to allow replication by others? Yes

If applicable, is the statistical analysis and its interpretation appropriate?

Partly

Are all the source data underlying the results available to ensure full reproducibility? Yes

Are the conclusions drawn adequately supported by the results?

Yes

Competing Interests: No competing interests were disclosed.

Reviewer Expertise: Diagnostics; statistics

I confirm that I have read this submission and believe that I have an appropriate level of expertise to confirm that it is of an acceptable scientific standard, however I have significant reservations, as outlined above.

Author Response 21 May 2022

Alexandra Cann

My only concern is the sample design and analysis of the HCW cohort. The calculations assume that the 78 samples are independent, but they are in fact pairs from 39 individuals measured before and after vaccination. I would sympathize with neglecting this issue for cases with a negative result at day 0 followed by a positive result at day 21 , which one might treat as 'sufficiently' independent; however, one is not surprised to find the 13 positive samples of day 0 to also test positive at day 21 . An ad-hoc remedy for this double-counting is to remove 13 of these 26 samples from the calculation as they provide no new information and artificially inflate the sample size. The estimated sensitivity is then reduced to $87.7 \%$ with a wider $95 \%$-CI of $[75.8,97.1]$ (compare to the reported $92.3 \%$ ) for that cohort, and to $91.0 \%(95 \%-C I[84.1,95.6])$ instead of $92.0 \%$ for the pooled analysis. Specificities are of course unaffected. The equally problematic case of negative results on both occasions does not occur in these data. Please comment on the sample design of the HCW cohort and its consequences. 
Many thanks for this comment. We acknowledge as a result of the pragmatic study design, some participants in the HCW cohort (13) have provided two samples to the study, at different time points ( $>21$ days apart), which have been treated as independent samples for the purpose of testing the accuracy of the LFIA devices against gold-standard. We agree that if this study was looking at individual differences in participants, it would not be appropriate to include both sets of samples as this would introduce bias. In individuals who have provided two samples, it is likely that both the quantitative and qualitative immune responses will be different. As such, given that the primary outcome for this study is to test the diagnostic accuracy of the LFIA device against the gold-standard Abbott titre we feel it is helpful to include these samples in the analysis.

Acknowledging the reviewer's comment, we have included a section addressing this in the discussion section and have shown the proposed sensitivity analysis removing the duplicate participants' samples' in the supplement.

A significance level of $15 \%$ is reported for the sample size calculation; statistical folklore and the reported sample size suggest it to mean $5 \%$.

This was a mistake and has been edited in the paper. The significance level is 5\%.

In Table 2, you report $\mathrm{N}=39$ for the D21 seropositive column, but males and females add to 40.

This has been changed.

Please add a label for the vertical axis of Figure 1.

Label added.

Competing Interests: No competing interests were disclosed.

Reviewer Report 01 February 2022

https://doi.org/10.21956/wellcomeopenres.19045.r47744

(c) 2022 Gupta-Wright A. This is an open access peer review report distributed under the terms of the Creative Commons Attribution License, which permits unrestricted use, distribution, and reproduction in any medium, provided the original work is properly cited.

\section{Ankur Gupta-Wright}

Institute for Global Health, University College London, London, UK

This study assesses the diagnostic accuracy of the Fortress LFIA for SARS-CoV-2 anti-S Ab compared to a lab-based serological assay reference standard. The study is appropriately conducted, based on two different cohorts but giving similar results. The authors have attempted to understand if FP results are due to FN results from the reference standard by using an additional assay for discordant results. 
Comments that need addressing:

Discussion

The small sample size leads to wide CIs - this needs to be emphasised in the discussion/limitations section.

Please state what the previously reported diagnostic accuracy for natural infection was for this assay.

Is the work clearly and accurately presented and does it cite the current literature?

Yes

Is the study design appropriate and is the work technically sound?

Yes

Are sufficient details of methods and analysis provided to allow replication by others?

Yes

If applicable, is the statistical analysis and its interpretation appropriate?

Yes

Are all the source data underlying the results available to ensure full reproducibility? Yes

Are the conclusions drawn adequately supported by the results?

Yes

Competing Interests: No competing interests were disclosed.

Reviewer Expertise: Diagnostics, Infectious Diseases

I confirm that I have read this submission and believe that I have an appropriate level of expertise to confirm that it is of an acceptable scientific standard.

Author Response 21 May 2022

\section{Alexandra Cann}

The small sample size leads to wide CIs - this needs to be emphasised in the discussion/limitations section.

Thank you for this comment. We have addressed this in the discussion/limitations section. Please state what the previously reported diagnostic accuracy for natural infection was for this assay.

We have included information and the reference paper on the reported diagnostic accuracy for natural infection for this assay. 
Competing Interests: No competing interests were disclosed. 